[Article]

\title{
高覆盖率氟代癸基三氯硅烷自组装单分子膜的制备
}

周家栋 董依慧 张帅辉 赵元元 郭小飞 陆小华 王昌松

(南京工业大学化工学院, 材料化学工程国家重点实验室, 南京 210009)

摘要: 通过液相沉积在云母表面制备 $1 H, 1 H, 2 H, 2 H$-全氟癸基三氯硅烷(FDTS)自组装单分子膜(SAMs)。室温 下, 将 $1.0 \mathrm{mmol} \cdot \mathrm{L}^{-1}$ 的 FDTS 溶液静置水解 $15 \mathrm{~min}$, 再把云母浸入自组装 $30 \mathrm{~min}$, 原子力显微镜(AFM)表征 发现, 液相沉积过程中FDTS 的团聚现象得到有效解决。该方法制备出了高覆盖率 $(85 \% \pm 2 \%)$ 和低均方根粗 粘度 $(0.58 \mathrm{~nm})$ 的 FDTS SAMs, 且单分子膜的生长过程符合 Langmuir一级动力学吸附模型。在液相沉积过 程中, 若水解和组装同时进行, 过长的水解时间(大于 $30 \mathrm{~min}$ )或组装时间(大于 $30 \mathrm{~min}$ )均会导致FDTS 的团 聚, 进而极大降低SAMs 的质量。

关键词: $1 H, 1 H, 2 H, 2 H$-全氟癸基三氯硅烷; 自组装单分子膜; 覆盖率; 团聚; 原子力显微镜 中图分类号: 0647

\section{Preparation of High-Coverage Fluorinated Decyltrichlorosilane Self-Assembled Monolayers}

\author{
ZHOU Jia-Dong DONG Yi-Hui ZHANG Shuai-Hui ZHAO Yuan-Yuan \\ GUO Xiao-Fei LU Xiao-Hua WANG Chang-Song*
}

(State Key Laboratory of Materials-Oriented Chemical Engineering, College of Chemical Engineering,

Nanjing University of Technology, Nanjing 210009, P. R. China)

\begin{abstract}
In this work, $1 \mathrm{H}, 1 \mathrm{H}, 2 \mathrm{H}, 2 \mathrm{H}$-perfluorodecyltrichlorosilane (FDTS) self-assembled monolayers (SAMs) were prepared using liquid-phase deposition. To prepare the FDTS SAMs, the FDTS solution was first hydrolyzed for $15 \mathrm{~min}$ at room temperature, and then mica was immersed into the FDTS solution for self-assembly for 30 min. It was confirmed that the new method can effectively prevent aggregation of FDTS in the liquid phase deposition using atomic force microscopy (AFM). The FDTS SAMs were successfully prepared with a high surface coverage of $(85 \% \pm 2 \%)$ and low root mean square surface roughness $(0.58 \mathrm{~nm})$. The growth process of the SAMs can be described using the Langmuir first-order adsorption kinetics model. However, if hydrolysis and assembly occur at the same time and these processes last more than $30 \mathrm{~min}$, the FDTS would be aggregated in the liquid-phase deposition process, which would greatly reduce the quality of the SAMs.
\end{abstract}

Key Words: $\quad 1 H, 1 H, 2 H, 2 H$-perfluorodecyltrichlorosilane; Self-assembled monolayer; Coverage; Aggregation; Atomic force microscope

1 引 言 近年来, 硅烷类自组装膜作为表面修饰层被
广泛用于微/纳米机电系统 ${ }^{1,2} 、$ 仿生材料 ${ }^{3} 、$ 生物传 感器 4 、腐蚀防护 5 等领域。其中 $1 H, 1 H, 2 H, 2 H$-全氟

Received: December 2, 2015; Revised: February 1, 2016; Published on Web: February 17, 2016.

*Corresponding author. Email: wcs@njut.edu.cn; Tel: +86-13951897581.

The project was supported by the National Key Basic Research Program of China (973) (2013CB733503), National Natural Science Foundation of China (21490581), and Project of Priority Academic Program Development of Jiangsu Higher Education Institutions, China (PAPD). 国家重点基础研究发展规划(973) (2013CB733503), 国家自然科学基金(21490581)和江苏高校优势学科建设工程(PAPD)资助项目 
癸基三氯硅烷(FDTS) 作为 $\mathrm{F}_{3} \mathrm{C}\left(\mathrm{CF}_{2}\right)_{m}\left(\mathrm{CH}_{2}\right)_{n} \mathrm{SiCl}_{3}$ 类氟 代有机硅烷, 由于氟原子的强电负性, 使得 FDTS 具有更低的表面能。同时头基 3 个 $\mathrm{Si}-\mathrm{Cl}$ 键理论上 通过吸附、水解、缩合可以在基底表面形成一层 结合牢固、致密有序的自组装单分子膜 $(\mathrm{SAMs})^{6-8}$, 因而可以赋予材料更高耐热性、自去 污、抗摩擦、抗粘附及抗菌性能 ${ }^{9-12}$ 。

液相沉积是制备 SAMs 的常用方法, 相比于 化学气相沉积法 ${ }^{13}$ 、电化学沉积法 ${ }^{14}$ 等, 其具有操 作简单、易于控制的特点。然而直接液相沉积易 造成 FDTS 在材料表面团聚, 从而严重降低了 FDTS 的使用性能。Kushmerick 等 ${ }^{15}$ 发现微机电系 统中团聚的FDTS 使自组装膜的覆盖率降低、粗 糙度增大, 不仅没有降低界面粘滞作用, 反而加 重了系统静摩擦。Bunker ${ }^{16}$ 和 $\operatorname{Tan}^{17}$ 等也提出了相 同观点。因此, 制备出高覆盖率、平整有序的 FDTS SAMs 对于实际应用具有重要的意义。针对 FDTS 的团聚问题, Bunker 等 ${ }^{16}$ 通过液相沉积发现 FDTS 呈液滴状团聚在硅片表面, 光散射实验证明 类似团聚体存在于溶液中, 溶剂中水含量是造成 团聚的重要因素; Pellerite 等 ${ }^{18}$ 认为高湿度环境中 的水可以破坏 SAMs 的有序结构; Tan 等 ${ }^{17}$ 研究发 现溶剂的溶解性在 FDTS SAMs 形成过程中扮演重 要作用, 与 FDTS 结构和极性相似的溶剂更易于 其成膜。Choi 等 ${ }^{19}$ 发现在类钻碳膜表面高浓度下 FDTS 团聚明显, 且随自组装时间延长, 团聚现象 加重。

上述文献从溶剂类型、溶剂水含量、环境湿 度、硅烷浓度等角度对 FDTS 的团聚问题进行了研 究, 但对自组装前溶液水解时间造成的影响未见 报道。因此, 本文针对液相沉积制备 FDTS SAMs 过程中的团聚问题。以云母为基底, 通过原子力 显微镜(AFM)探究不同水解和组装时间对FDTS 成 膜的影响, 从而制备出高覆盖的 SAMs。同时本文 对FDTS 形成SAMs 与团聚的机制进行了探讨。

\section{2 实验部分}

\section{1 实验药品及仪器}

FDTS (96\%)购于 TCI 公司(日本), 并未处理直 接使用。异辛烷 $(90.0 \%$, 含水量 $<0.01 \%)$ 、丙酮 (99.6\%)、四氯化碳 $(99.5 \%)$ 、无水乙醇 $(99.7 \%)$ )、异 丙醇 $(99.7 \%$ )均为分析纯级试剂(购于国药试剂集 团)。手套箱(伊特克斯公司, Lab2000)、微量移液
枪、云母片(中镜科仪)。

\section{2 样品的制备}

向密闭的手套箱中通入氮气, 保持箱内湿度 为 $8 \%-10 \%$, 温度 $(20 \pm 2){ }^{\circ} \mathrm{C}$ 。以异辛烷为溶剂, 配置 $1.0 \mathrm{mmol} \cdot \mathrm{L}^{-1}$ 的 FDTS 溶液。静置水解数分 钟, 然后将新解理的云母立即投入到溶液中组装 一定的时间取出。分别用丙酮, 四氯化碳, 异丙 醇于超声条件下各清洗 $1 \mathrm{~min}$, 以除去表面多余 的 FDTS 分子。然后用高纯氮气吹干, 于 $120^{\circ} \mathrm{C}$ 的无尘烘箱中热固化 $1 \mathrm{~h}$, 待冷却至常温立即进 行表征。

\section{3 样品表征}

采用原子力显微镜(布鲁克 Dimension Icon)对 云母表面的 SAMs 进行表征, 所有样品测试均在室 温大气环境中进行。扫描模式为 PeakForce Tapping, 选用三角形悬臂探针, 探针为氮化硅材质, 悬臂弹性系数 $0.4 \mathrm{~N} \cdot \mathrm{m}^{-1}$, 扫描图像分辨率 $256 \times$ 256 , 扫描速率 $1.0 \mathrm{~Hz}$ 。

利用 Nanoscope Analysis 离线分析软件来得到 测试后 SAMs 的表面形貌、高度、覆盖率 $(\theta)$ 、均 方根粗䊁度 $\left(R_{\mathrm{q}}\right)$ 值。其中 $\theta$ 的计算采用软件中的 “Bearing Analysis” 功能实现, 以 $0.2 \mathrm{~nm}$ (云母均高 度)为界来区分基底和 $\mathrm{SAMs}^{20}$ 。 $R_{\mathrm{q}}$ 采用 “Roughness” 功能实现, 用来表示膜的有序程度 ${ }^{21}$ (本文的 $R_{\mathrm{q}}$ 均为相对于 $2 \mu \mathrm{m} \times 2 \mu \mathrm{m}$ 的结果)。实验对不同水 解和组装时间各进行了 3 批次的测试, 同时对各批 次中每个样品多点扫描, 以避免因局部误差而影 响统计结果的可靠性。

\section{3 结果与讨论}

\section{1 水解时间对 SAMs 制备的影响}

图 1 表示水解不同时间后云母表面 FDTS 自组 装膜的 AFM 形貌及相应的高度图, 图中同时给出 了不同时间下的 $\theta$ 和 $R_{\mathrm{q}}$ 值。图 1(a)中自组装膜呈颗 粒状吸附在云母表面, 自组装膜分布稀疏, $\theta$ 仅 $10 \%$ 左右; 高度图看出膜的平均高度大于 FDTS 分 子理论高度 $1.5 \mathrm{~nm}^{16}$, 其中最大高度可达 $23 \mathrm{~nm}$ 。 Kushmerick $^{15}$ 和 Raza ${ }^{7}$ 等通过直接液相沉积在硅片表 面得到类似的团聚形貌。

图1(c)显示自组装膜呈碎屑状吸附在云母表 面且分布非常均匀。膜的高度在 $0.6-1.5 \mathrm{~nm}$ 之 间, 其中岛状高度为 $1.5 \mathrm{~nm}$, 而岛周围的点状高 度则低于 $1.5 \mathrm{~nm}$ 。说明此时自组装膜同时存在直 

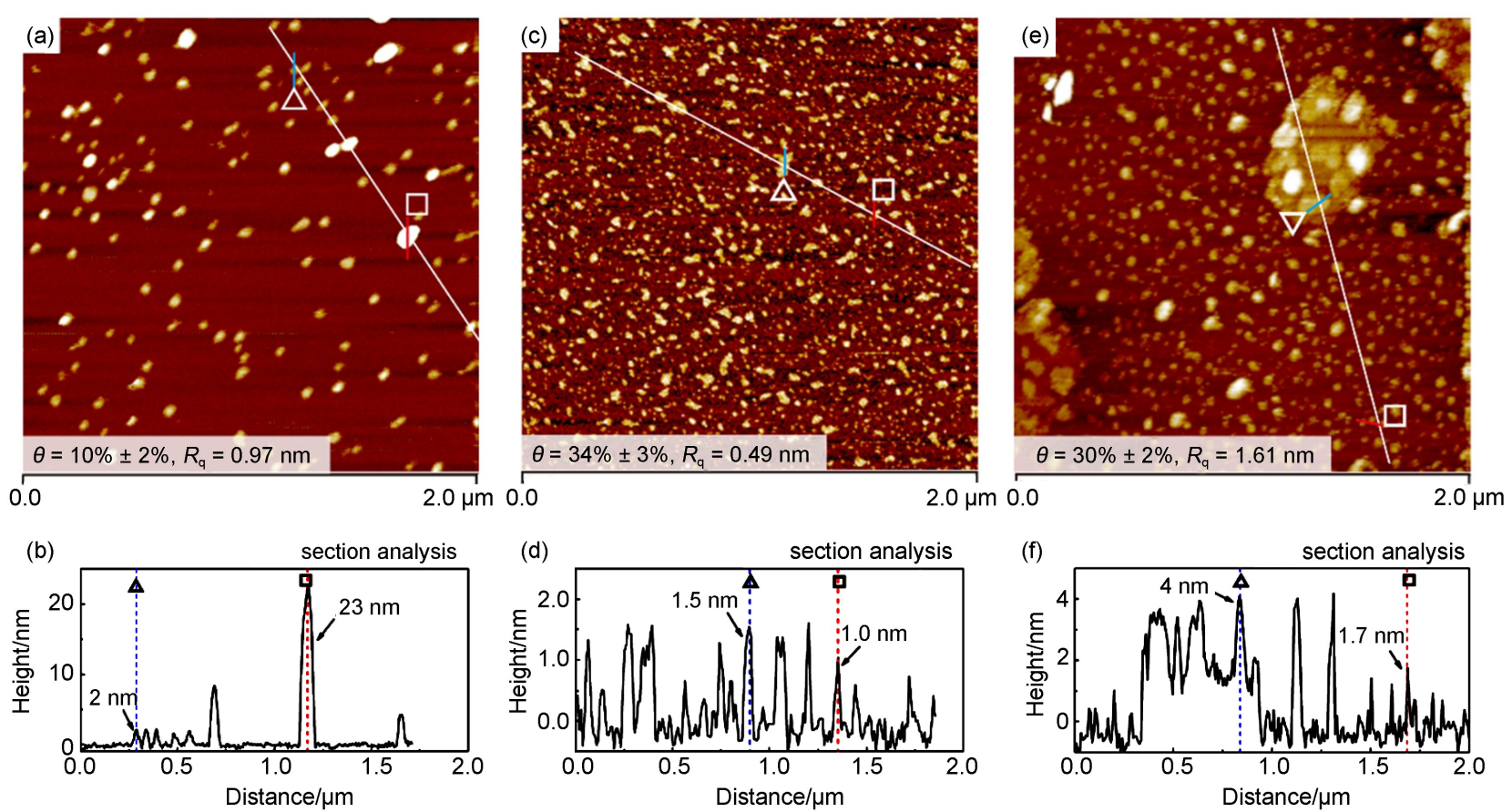

图 1 水解不同时间后云母表面 FDTS 自组装膜的 AFM 二维形貌及相应的高度图

Fig.1 Two-dimensional AFM morphologies and the section height profile of FDTS self-assembled films on mica at different hydrolysis time

(a, b) $0 \mathrm{~min},(\mathrm{c}, \mathrm{d}) 15 \mathrm{~min},(\mathrm{e}, \mathrm{f}) 60 \mathrm{~min}$. The coverage of SAMs $(\theta)$ and root mean square surface roughness $\left(R_{\mathrm{q}}\right)$ are shown in the figures.

立和倾斜两种状态。与图 1(a)相比，静置水解 15 $\min$ 形成自组装膜 $\theta$ 增加了近 3 倍, $R_{\mathrm{q}}$ 降低了一 半，自组装膜的质量明显提高。图 1(e)自组装膜 同样出现颗粒状团聚的现象。与图 1(a)不同, 水 解 $60 \mathrm{~min}$ 后形成团聚物明显较大, 其高度在 $4 \mathrm{~nm}$ 左右。与图 1(c)相比, 膜的覆盖率相近, 但粗糙 度增大了近 3 倍。

上述结果表明, 水解时间对 FDTS SAMs 影响 非常明显。恰当的水解时间可以有效的降低 FDTS 的团聚问题, 明显提高 SAMs 的制备质量。通过单 因素实验得到当前实验条件下溶液的最佳水解时 间为 $15 \mathrm{~min}$, 水解超过 $30 \mathrm{~min}$ 就会出现明显团聚 现象。

\section{2 组装时间对 SAMs 制备的影响}

图 2 是将云母在水解 $15 \mathrm{~min}$ 后的FDTS 溶液中 组装不同时间得到自组装膜的 AFM 形貌及高度 图。由图 2(a-d)的三维形貌图可以看出, 自组装 膜呈簇状吸附在云母表面。随组装时间增加, 簇 状膜的吸附量逐渐增加。从高度图变化看出, 吸 附岛高度逐步趋于 $1.5 \mathrm{~nm}$, 簇状膜的间距逐渐变 小, 膜的有序程度和致密程度增加。组装 $30 \mathrm{~min}$ 时(图 2(d)), 自组装膜呈单分子层结构, 覆盖率达
到 $85 \% \pm 2 \%$, 粗糙度为 $0.58 \mathrm{~nm}$ (具有原子级平整 度的云母 $R_{\mathrm{q}}$ 约为 $0.20 \mathrm{~nm}$ )。二维形貌图中看出此时 SAMs 呈沟壑状分布。当继续延长组装时间至 40 $\min ($ 图 2(e)), SAMs 表面会出现非均一的无序结 构, 其高度可达到 $3.5 \mathrm{~nm}$, 粗糙度增大到 0.94 $\mathrm{nm}$, 此时自组装膜质量明显变差。

图 3 是 $30 \mathrm{~min}$ 内云母表面 FDTS SAMs 覆盖率 随组装时间的变化曲线。图中看出随浸泡时间增 加, 覆盖率先迅速增加后趋于缓慢。文献 ${ }^{22}$ 中对于 SAMs 的生长过程用 Langmuir 一级动力学吸附模型 进行过描述:

$$
\theta / \theta_{0}=1-\mathrm{e}^{-K C t}
$$

其中 $\theta$ 是 $t$ 时间的覆盖率, $\theta_{0}$ 为最大覆盖率, $K$ 为吸 附速率常数, $C$ 为 FDTS 组装液浓度 $(1 \mathrm{mmol}$. $\left.\mathrm{L}^{-1}\right)$ 。通过对 $-\ln \left(1-\theta / \theta_{0}\right)$ 与 $t$ 线性拟合得到 $K=$ $0.153 \mathrm{~min} \cdot \mathrm{L} \cdot \mathrm{mmol}^{-1}$, 其中线性相关度 $R^{2}=0.986$, 说明 FDTS 分子在云母表面吸附符合 Langmuir 一级 吸附模型。

\subsection{FDTS SAMs 成膜机理讨论}

图 4 将上述实验工作分为四组进行形成机理讨 论：(a) 水解和组装同时进行 (直接液相沉积); (b) 先水解 $15 \mathrm{~min}$ 再组装 $30 \mathrm{~min}$; (c) 先水解再长时间 

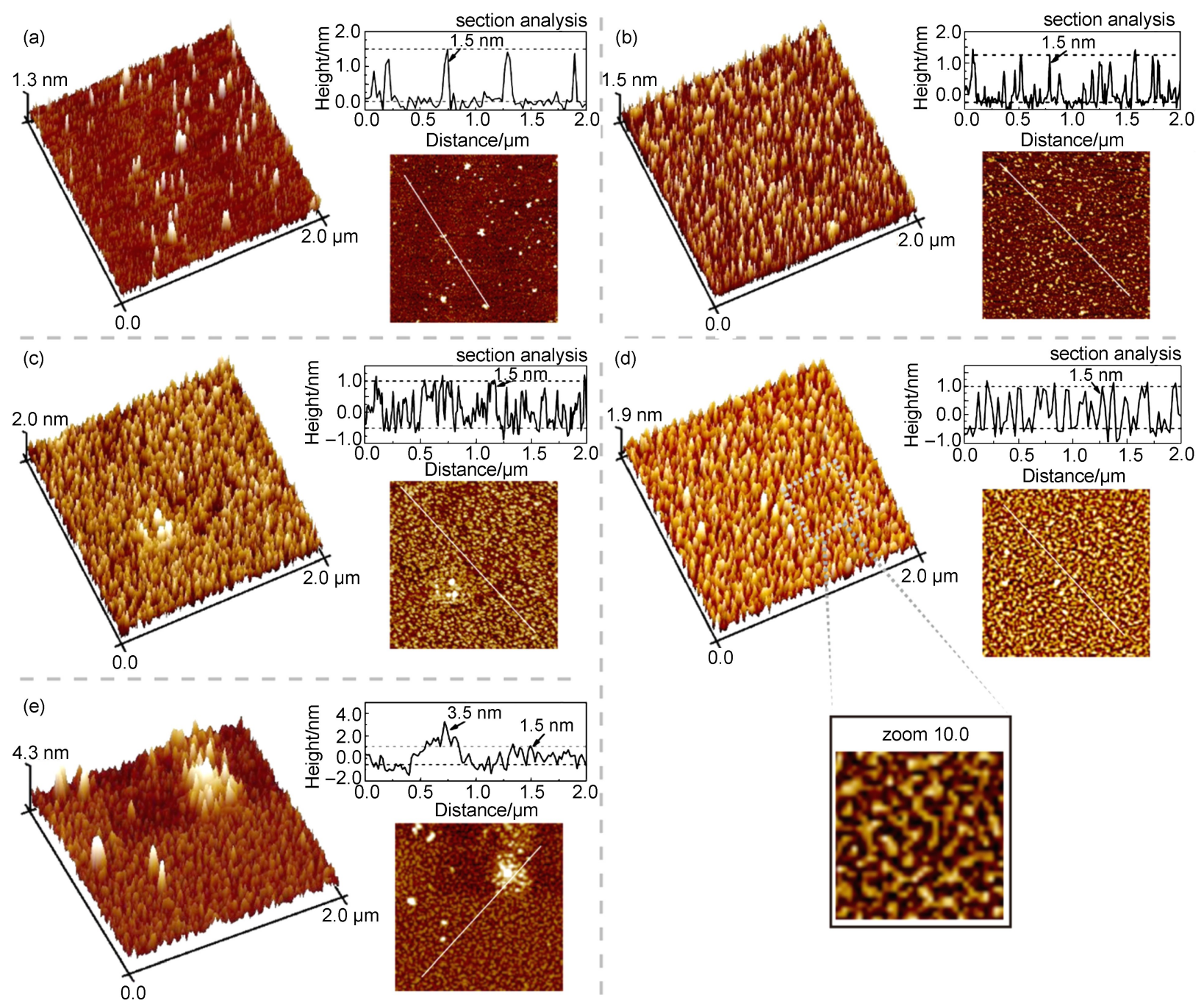

图 2 组装不同时间后云母表面 FDTS 自组装膜的 AFM 二维、三维形貌及相应的高度图

Fig.2 Two-dimensional, three-dimensional AFM morphologies, and the section height profile of FDTS self-assembled films on mica at different assembly time

(a) $1 \mathrm{~min}$, (b) $5 \mathrm{~min}$, (c) $15 \mathrm{~min}$, (d) $30 \mathrm{~min}$, (e) $40 \mathrm{~min}$

组装 $(>30 \mathrm{~min}) ;$ (d) 长时间水解 $(>30 \mathrm{~min})$ 再组 装。图 4(a)为直接液相沉积过程, FDTS 水解形成 硅醇单体会直接吸附到云母表面, 此时硅醇嫁接 密度低, 其只能倾斜吸附在表面。而单个硅醇分 子间范德华力较弱, 硅醇单体之间难以通过范德 华力 (VDW)相互靠近形成有序的膜。因而溶液中 多余的硅醇通过氢键作用结合到已吸附单体的羟 基上, 造成FDTS 团聚的形成。这种团聚体的形成 会进一步限制 FDTS 在基底表面的扩散和铺展成 膜 ${ }^{16}$, 并使团聚不断加重, 进而导致膜的低覆盖率 和高粗粘度(见图 1(a))。相比于目前研究比较成熟 的十八烷基三氯硅烷(OTS), OTS 通过直接液相沉 积可以形成有序、致密的 $\mathrm{SAMs}^{23-26}$ 。分析其原因, 硅烷分子链越长会导致有利于分子有序排列的范
德华力越大, 形成的单分子膜越致密有序 ${ }^{27}$ 。 FDTS 分子结构中只有 10 个 $\mathrm{C}$ 原子, 分子间的范德 华力明显低于含有 18 个 $\mathrm{C}$ 的 OTS。同时, 氟原子 体积较氢原子大, 使氟化碳链的排列密度降低, 碳链的自由度增加 ${ }^{28}$ 。因此, FDTS 通过直接液相 沉积难以形成高覆盖率 SAMs。

图 4(b) 是高覆盖率 SAMs 的形成过程。FDTS 在溶液水解过程中会通过头基的 $\mathrm{Si}-\mathrm{OH}$ 缩合形成 的硅醇低聚物。Vallant 等 ${ }^{29}$ 研究 OTS SAMs 时提出 相同的观点。而硅醇低聚物吸附到基底活性位点 速率快于硅醇单体 ${ }^{30}$, 因而当云母浸入组装液中 后, 硅醇低聚物会优先迅速吸附到基底上。根据 扩散限制凝聚理论 ${ }^{31,32}$, 具有较大范德华力的硅醇 低聚物, 会诱导硅醇单体发生迁移, 并与已吸附 


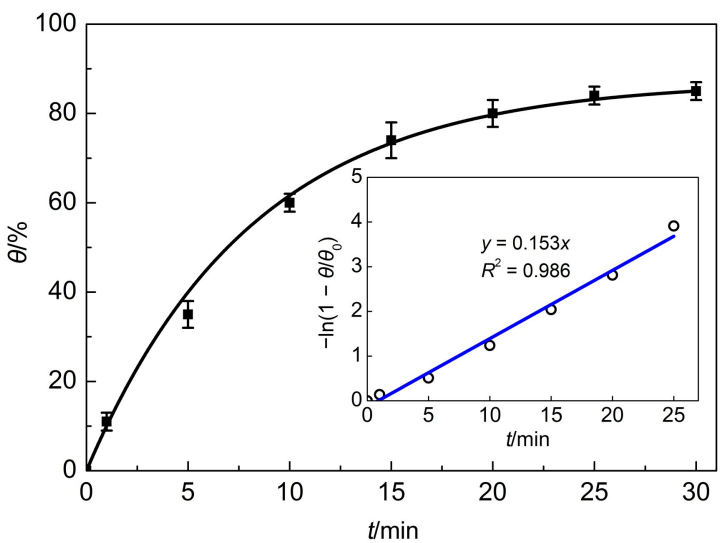

图 3 FDTS SAMs 形成过程中云母表面覆盖率与 组装时间的关系图

Fig.3 Relationship between coverage and assembly time during the formation processes of FDTS SAMs

The solid line are the best curve fit obtained with exponential functions

(Eq.(1)). From the logarithmic plot $-\ln \left(1-\theta / \theta_{0}\right)$ versus time,

the adsorption rate constant was determined as $K=0.153 \mathrm{~min} \cdot \mathrm{L} \cdot$ $\mathrm{mmol}^{-1} \cdot \theta$ is the coverage of SAMs at different assembly time $(t)$,

$\theta_{0}$ is the maximum coverage.
的硅醇低聚物发生缩合。通过水解、吸附、迁移 和缩合过程, FDTS 自组装膜不断增长, 最终形成 如图 2(d)的沟壑状单分子层形貌。

在图 4(c)过程中, 组装 $30 \mathrm{~min}$ 后的 SAMs 覆盖 率并未达到 $100 \%$ ，膜间存在一定的空隙。过长的 组装时间(>30 min)使得溶液中多余的硅醇低聚物 继续结合到空隙间的 $\mathrm{Si}-\mathrm{OH}$ 键上，从而使膜的粗 糙度增大 ${ }^{8,33}$ (如图 2(e) 所示)。在图 4(d)过程中，较 长的水解时间(>30 min)使溶液中水解形成的硅醇 浓度会增大，硅醇间彼此不断地交联，进而会形 成无序的高聚物吸附在基底表面。Bunker等 ${ }^{16}$ 认为 这种高聚物可能是反相胶束或多层膜结构。高聚 物的形成降低了自组装膜的有序度，同时严重增 大膜的粗糙度(如图 1(e)所示)。

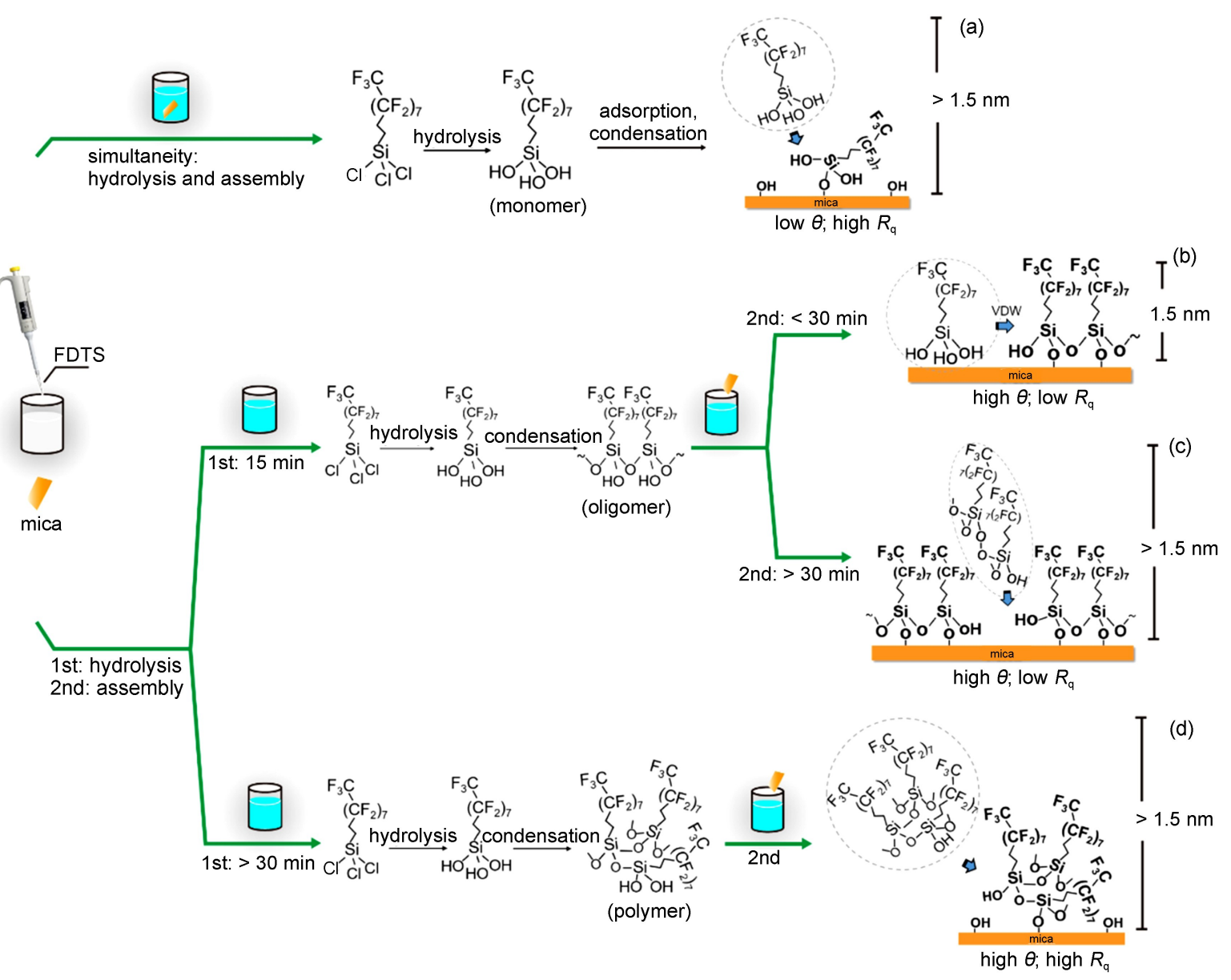

图 4 不同水解(第一步)和组装(第二步)时间下 FDTS 自组装膜的形成机理

Fig.4 Scheme of formation mechanism of FDTS self-assembled films under different hydrolysis time (1st) and assembly time (2nd)

(a) 1st and 2nd simultaneously; (b) 1 st $15 \mathrm{~min}$, 2nd $30 \mathrm{~min}$; (c) 1 st $15 \mathrm{~min}$, 2nd $>30 \mathrm{~min}$; (d) 1st $>30$ min then 2nd; VDW: van der Waals force 


\section{4 结 论}

在室温下, 将 $1.0 \mathrm{mmol} \cdot \mathrm{L}^{-1}$ 的 FDTS 溶液静置 水解 $15 \mathrm{~min}$, 再把云母浸入自组装 $30 \mathrm{~min}$ 的方 法, 有效降低了 FDTS 液相沉积过程中 SAMs 的团 聚问题, 成功制备出覆盖率为 $85 \% \pm 2 \%$, 均方根 粗䊁度为 $0.58 \mathrm{~nm}$ 的高质量FDTS SAMs, 且单分子 膜的生长过程符合 Langmuir 一级动力学吸附模 型。在 FDTS 自组装过程中, 水解和组装同时进 行, 过长的水解(大于 $30 \mathrm{~min}$ )或组装时间(大于 30 min)均会导致FDTS 的团聚, 难以制备出高质量的 SAMs。本文改善FDTS SAMs 质量将对材料表面 抗污染、抗摩擦和抗黏附研究提供重要基础。

\section{References}

(1) Pu, J. B.; Jiang, D.; Mo, Y. F.; Wang, L. P.; Xue, Q. J. Surface \& Coatings Technology 2011, 205, 4855. doi: 10.1016/j. surfcoat.2011.04.089

(2) Ewers, B. W.; Batteas, J. D. RSC Advances 2014, 4, 16803. doi: $10.1039 /$ C4RA01427H

(3) Xu, L. H.; Wang, L. M.; Shen, Y.; Ding, Y.; Cai, Z. S. Fibers and Polymers 2015, 16, 1082. doi: 10.1007/s12221-015-1082-x

(4) Wilson, K. A.; Finch, C. A.; Anderson, P.; Vollmer, F.; Hickman, J. J. Biomaterials 2012, 33, 225. doi: 10.1016/j. biomaterials.2011.09.036

(5) Wang, D.; Ni, Y.; Huo, Q.; Tallman, D. E. Thin Solid Films 2005, 471 (1-2), 177. doi: 10.1016/j.tsf.2004.06.158

(6) Desbief, S.; Patrone, L.; Goguenheim, D.; Guérin, D.; Vuillaume, D. Phys. Chem. Chem. Phys. 2011, 13, 2870. doi: 10.1039/C0CP01382J

(7) Raza, M. A.; Kooij, E. S.; van Silfhout, A.; Poelsema, B. Langmuir 2010, 26, 12962. doi: 10.1021/la101867z

(8) Kristalyn, C. B.; Watt, S.; Spanninga, S. A.; Barnard, R. A.; Nguyen, K.; Chen, Z. Journal of Colloid and Interface Science 2011, 353, 322. doi: 10.1016/j.jcis.2010.09.057

(9) Ling, F. Z.; De Coster, J.; Lin, W. Y.; Witvrouw, A.; Celis, J. P.; De Wolf, I. Sensors and Actuators A-Physical 2012, 188, 320. doi: 10.1016/j.sna.2012.01.011

(10) Frechette, J.; Maboudian, R.; Carraro, C. Journal of Microelectromechanical Systems 2006, 15, 737. doi: 10.1109/ JMEMS.2006.878893

(11) Hoque, E.; DeRose, J. A.; Hoffmann, P.; Mathieu, H. J. Surface and Interface Analysis 2006, 38, 62. doi: 10.1002/sia.2179

(12) Pan, Z. H.; Zhang, W.; Kowalski, A.; Zhao, B. X. Langmuir 2015, 31, 9901. doi: 10.1021/acs.langmuir.5b02884

(13) Wang, A.; Cao, T.; Tang, H.; Liang, X.; Black, C.; Salley, S. O.; Mcallister, J. P.; Auner, G. W.; Ng, K. Y. S. Colloids \& Surfaces B: Biointerfaces 2006, 47, 57. doi: 10.1016/j. colsurfb.2005.11.016

(14) Hu, J. M.; Liu, L.; Zhang, J. Q.; Cao, C. N. Progress in
Organic Coatings 2007, 58, 265. doi: 10.1016/j.

porgcoat.2006.11.008

(15) Kushmerick, J. G.; Hankins, M. G.; de Boer, M. P.; Clews, P. J.; Carpick, R. W.; Bunker, B. C. Tribology Letters 2001, 10, 103. doi: 10.1023/A:1009082530479

(16) Bunker, B. C.; Carpick, R. W.; Assink, R. A.; Thomas, M. L.; Hankins, M. G.; Voigt, J. A.; Sipola, D.; de Boer, M. P.; Gulley, G. L. Langmuir 2000, 16, 7742. doi: 10.1021/ la000502q

(17) Tan, M.; Zhang, H.; Wang, Y.; Ma, H.; Zhu, J.; Han, J. Applied Surface Science 2008, 254, 6332. doi: 10.1016/j. apsusc.2008.03.174

(18) Pellerite, M. J.; Wood, E. J.; Jones, V. W. Journal of Physical Chemistry B 2002, 106, 4746. doi: 10.1021/jp013820m

(19) Choi, J.; Sakurai, K.; Kato, T. Surface and Interface Analysis 2010, 42, 1373. doi: 10.1002/sia.v42:6/7

(20) Rozlosnik, N.; Gerstenberg, M. C.; Larsen, N. B. Langmuir 2003, 19, 1182. doi: 10.1021/1a025906s

(21) Balgar, T.; Bautista, R.; Hartmann, N.; Hasselbrink, E. Surface Science 2003, 532-535, 963. doi: 10.1016/S0039-6028

(22) Major, R. C.; Zhu, X. Y. Langmuir 2001, 17, 5576. doi: 10.1021/la010821x

(23) Carraro, C.; Yauw, O. W.; Sung, M. M.; Maboudian, R. Journal of Physical Chemistry B 1998, 102, 4441. doi: 10.1016/jp9810195

(24) Iimura, K. I.; Nakajima, Y.; Kato, T. Thin Solid Films 2000, 379, 230. doi: 10.1016/S0040-6090(00) 01544-3

(25) Wang, C. S.; Feng, X.; Lu, X. H. Acta Phys. -Chim. Sin. 2005, 21, 586. [王昌松, 冯 新, 陆小华. 物理化学学报, 2005, 21, 586.] doi: 10.3866/PKU.WHXB20050602

(26) Zhou, Z. W.; Xu, G. H.; An, Y.; He, C. H. Acta Phys. -Chim. Sin. 2014, 30, 1509. [朱志文, 徐国华, 安 越, 何潮洪. 物理 化学学报, 2014, 30, 1509.] doi: 10.3866/PKU. WHXB201405282

(27) Stevens, M. J. Langmuir 1999, 15, 2773. doi: 10.1021/ la981064e

(28) Robinson, G. N.; Kebabian, P. L.; Freedman, A.; Depalma, V. Thin Solid Films 1997, 310, 24. doi: 10.1016/S0040-6090(97) 00327-1

(29) Vallant, T.; Brunner, H.; Mayer, U.; Hoffmann, H.; Leitner, T.; Resch, R.; Friedbacher, G. Journal of Physical Chemistry B 1998, 102, 7190. doi: 10.1021/jp981282g

(30) Resch, R.; Grasserbauer, M.; Friedbacher, G.; Vallant, T.; Brunner, H.; Mayer, U.; Hoffmann, H. Applied Surface Science 1999, 140, 168. doi: 10.1016/S0169-4332(98) 00585-6

(31) Witten, T. A.; Sander, L. M. Physical Review Letters 1981, 47, 1400. doi: 10.1103/PhysRevLett.47.1400

(32) Meakin, P. Physical Review Letters 1983, 51, 1119. doi: 10.1103/PhysRevLett.51.1119

(33) Paso, K.; Helberg, R. M. L.; Raaen, S.; Sjöblom, J. Journal of Colloid \& Interface Science 2008, 325, 228. doi: 10.1016/j. jcis.2008.05.015 